\title{
Halving and doubling isometric force: Evidence for a decelerating psychophysical function consistent with an equilibrium-point model of motor control
}

\author{
CLAYTON L. VAN DOREN \\ Case Western Reserve University, Cleveland, Ohio
}

\begin{abstract}
Several previous investigations have measured accelerating psychophysical functions for perceived force with exponents of about 1.7. Two halving and doubling experiments presented here imply a psychophysical function for perceived force with an exponent between 0.6 and 0.8 . That is, more than a doubling of force was needed to double the sensation, and similarly for halving. In the first experiment, subjects squeezed rigid instrumented cylinders between the thumb and first two fingers of each hand. They generated and released a reference force with one hand, and then squeezed the opposite hand to produce a sensation magnitude equal to, twice that, or half that of the reference. An analysis using a model that accounted for compression bias yielded average psychophysical functions with exponents of 0.58 and 0.59 (nondominant and dominant hands, respectively). The second experiment was an attempt to replicate earlier results and to reconcile them with the first experiment by using a paradigm duplicated from a previous study. Subjects in the second experiment made unilateral halving and doubling judgments of handgrip while squeezing a hand dynamometer. Again, the halving and doubling judgments yielded decelerating functions with exponents of 0.75 and 0.80 (nondominant and dominant hands, respectively). Even though the results of the first two experiments contradict earlier investigations, they can be explained by an equilibrium model of motor control assuming that subjects halve and double the central motor command rather than the sensation of force. The force is simply the result of the mechanical equilibrium established between the load and the compliant effector (the hand). The predicted relationship between the motor command judgments, the compliance of the hand, and the resultant forces was confirmed in a third experiment in which the mechanical compliance of the three-finger pinch was measured by using a pneumatic manipulandum to apply force perturbations in a "do-notintervene" paradigm. The measured compliance characteristic was accelerating, just as predicted by the model, in order to produce a decelerating psychophysical function for "perceived force." In this experiment, then, judgments of perceived force appear to be judgments of the central motor command.
\end{abstract}

The perception of the force produced by muscular effort has presented a puzzle to psychologists and physiologists since the early nineteenth century (for a historical review, see Jones, 1986). Unlike other sensory continua, such as brightness, sweetness, or loudness, force "stimuli" are both perceived and created by the subject. The intriguing question is whether persons make "force" judgments on the basis of the afferent information arising from muscular, articular, and cutaneous receptors, or instead judge some central variable related to the efferent motor command (for reviews, see Gandevia \& Burke, 1992; Jones, 1986; Matthews, 1982).

There is evidence for both types of judgments. Judgments based on the efferent command, often referred to as per-

This research was supported by Grant NS-2-7958 from the National Institutes of Health. The author would like to thank three anonymous reviewers for helpful comments on earlier versions of this manuscript, and Purwanto Suwondo, Jennifer Bippus, and Scott Heavner for excellent technical assistance. The author's mailing address is Case Western Reserve University, MetroHealth Medical Center, H601, 2500 MetroHealth Dr., Cleveland, OH 44109-1998 (e-mail: clv2@po.cwru.edu). ceived effort, are characterized by a dependence on the strength of the muscle at the time of the judgment. Cafarelli and Bigland-Ritchie (1979), for example, used a bilateral matching paradigm and showed that if the maximum voluntary contraction (MVC) is reduced by shortening a muscle, the perceived effort at a constant force increases roughly in inverse proportion to the decreasing MVC. Gandevia and McCloskey (1977), likewise, showed that weakening muscles by partial curarization increases the perceived effort at a given actual force. Muscular fatigue has a similar effect. As the fatigued muscle gets weaker, greater effort is required to maintain a constant force, yielding increased magnitude estimates of force (J. C. Stevens \& Cain, 1970) or increased force matches made with the nonfatigued limb (Jones \& Hunter, 1983a, 1983b). Jones and Hunter (1983a) found that fatigue-induced matching errors persist even if the subjects are instructed to match the actual force and ignore changes in effort.

Roland and Ladegaard-Pedersen (1977), in contrast, showed that subjects can make bona fide force judgments if instructed to do so. Gallamine was used to induce paresis in one arm, and subjects who were instructed specifi- 
cally to match forces between the paretic and control arm did so without systematic errors. Subjects instructed to match effort produced larger forces with the control arm than with the paretic arm, roughly in proportion to the ratio of the normal to paretic MVCs. A similar distinction between instructions to match effort or match tension (against a strain gauge) resulted in variable or constant forces, respectively, during vibrations applied to the tendon (McCloskey, Ebeling, \& Goodwin, 1974).

Finally, Eisler (1962) obtained results consistent with both effort and force matching with the same subjects and apparatus - and, presumably, with the same instructions to judge "effort" or "force"-but different matching paradigms. Subjects matched handgrip force to leg-press force either directly or - by matching both grip and leg press to white noise first and then (mathematically) equating forces matched to the same sound pressure-indirectly. The forces were proportional to each other for both direct and indirect matches. Handgrip force was only $22 \%$ of the leg-press force when grips were matched to leg presses, consistent with effort matching. That is, at equal efforts, the leg should produce substantially more force than the hand. Grip force was $63 \%$ of leg-press force, however, when leg presses were matched with handgrip. And most surprisingly, grip force was $85 \%$ of leg-press force when both were matched to equal levels of white noise, as if the subjects were (nearly) making judgments of actual force.

Given the potential for afferent- or efferent-based matching judgments and the dependence on specific instructions and paradigms, it is surprising, perhaps, that psychophysical scaling functions for perceived force or effort appear to be quite stable across a wide variety of effortful activities. J. C. Stevens $(1974,1989)$ cites seven different experiments that measured psychophysical functions for isometric force with exponents that ranged from 1.5 to 1.8. ${ }^{1}$ The constancy over measurement method (magnitude estimation and production, halving and doubling, crossmodality matching) and task (handgrip, arm curls, biting, and leg presses) prompted J. C. Stevens (1974) to call the function a psychophysical "invariant."

The invariant is not without a few exceptions, however. As reviewed by Jones (1986), three studies (Banister, 1979; Cooper, Grimby, Jones, \& Edwards, 1979; Jones \& Hunter, 1982) measured linear or decelerating psychophysical functions, but the forces were estimated or produced relative to the MVC. Geometric means of the production and estimation exponents for judgments made as "percent of maximum effort" (Cooper et al., 1979) were 0.98 and 0.85 for isometric contractions of the adductor pollicis and quadriceps, respectively. Jones and Hunter (1982) obtained magnitude-production exponents of "perceived force" (using integers from 1 to 10 , with 10 assigned to the MVC) that ranged from 0.45 to 0.86 across subjects for isometric flexion contractions of the long finger.

It is possible that the difference between the two sets of scaling results is due to differences in the scaling techniques (Poulton, 1989). Cooper et al. (1979, p. 179) suggested that assignment of the maximum rating to the MVC might "inadvertently depress the rating of force near maximum forces" (although Eisler, 1965, found no such effect for leg presses up to the subject's maximum). It is also well known that a modulus can induce spurious curvature in its vicinity (Hellman \& Zwislocki, 1961). The scaling functions would be distorted also if the subjects resorted to categorization rather than ratio scaling (S. S. Stevens \& Galanter, 1957; J. C. Stevens \& Mack, 1959).

In the experiments presented here, an attempt was made to avoid ceiling effects or potential biases from categorization by asking subjects to make halving-doubling judgments without reference to their MVC (which was not measured until after the experiments were complete). Even so, the first experiment yielded a decelerating psychophysical function (exponent of 0.6 ) for bilateral halving and doubling of three-finger pinch force, in contrast to earlier halvingdoubling results (Bernyer, 1957, as cited in J. C. Stevens, 1974; J. C. Stevens \& Mack, 1959). To test whether the small exponent was simply due to methodological differences, the second experiment repeated the halving-doubling judgments but used handgrip and a unimanual protocol that was nearly identical to that used by J. C. Stevens and Mack (1959). Again, the matching ratios were consistent with a decelerating psychophysical function.

The reason for the difference between the present and previous results remains elusive, but it may arise from the afferent-efferent duality of kinesthetic perception. It is possible to provide a cohesive explanation for the present halving-doubling results by linking the psychophysical judgments to motor control. An equilibrium-point model is described (after Feldman \& Latash, 1982) that accounts for both the perception and production of force by postulating that subjects halved or doubled the central control variable (efferent command) rather than afferent-based perceptions of force. The model's predictions were tested in a third experiment by measuring the mechanical stiffness of the hand in a three-finger pinch using force perturbations in a "do-not-intervene" paradigm. The measured stiffnesses agreed with the simultaneous requirements of the equilibrium-point model and ratio judgments.

\section{EXPERIMENT 1 Bilateral Halving and Doubling}

\section{Method}

Subjects. Two female and 5 male subjects, 21 to 26 years old and with no reported sensorimotor disorders, participated in the experiment. Six subjects were right-handed.

Apparatus. In each session, the subject sat in a chair with arms at his/her sides, elbows flexed about $90^{\circ}$, and forearms supported at a comfortable level by small foam-cushioned tables. The subject's hands extended past the end of the tables, and held a manipulandum between the thumb and first two fingers of each hand. Each manipulandum, as shown in Figure 1, was a rigid cylinder instrumented with a miniature compression load cell. Each manipulandum weighed $52 \mathrm{~g}$ and was suspended from the ceiling by a long, compliant spring that allowed the subject to grasp it lightly without fear of dropping it (Van Doren, 1993). The suspension springs were very compliant (stiffness approximately $0.012 \mathrm{~N} / \mathrm{cm}$ ), so the thumb and finger forces would be nearly balanced over modest ranges of hand displacements. Both manipulanda were fully calibrated prior to the experiment, and the calibrations were checked prior to each session. The 


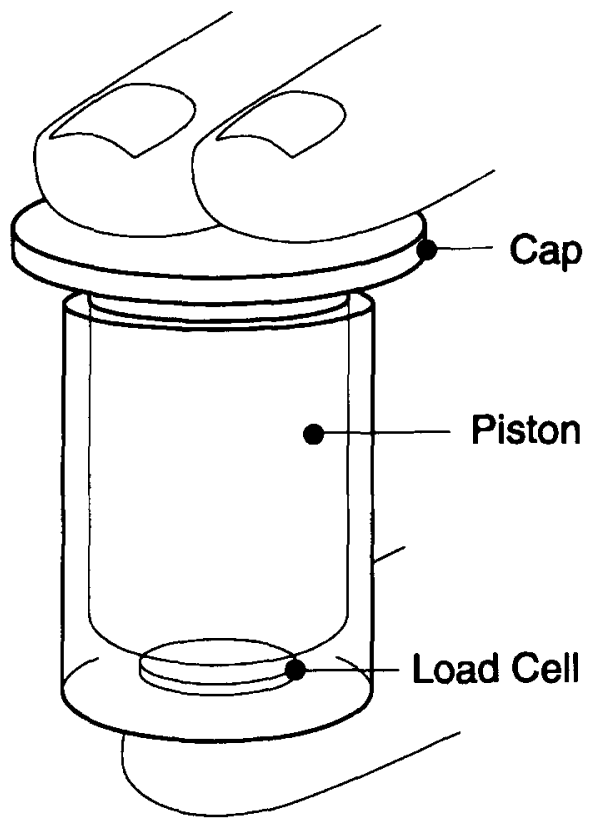

Figure 1. The manipulanda in Experiment 1 were rigid cylinders, $4.6 \mathrm{~cm}$ long and, at the bottom, $2.5 \mathrm{~cm}$ wide (thumb contact), with a 3.3-cm cap to contact the fingers. The cap was connected to an inner piston that compressed a miniature load cell. The cylinders weighed $52 \mathrm{~g}$.

overall sensitivity of the system was about $25 \mathrm{mV} / \mathrm{N}$. The force signal was sampled at $50 \mathrm{~Hz}$ for each cylinder using an analog $\mathrm{V} / \mathrm{O}$ board and microcomputer.

Procedure. General instructions were read to each subject prior to every session. The subjects were instructed to match the "perceptions of pinch force" between their hands and to make pinches with their matching hands "so that the size of the sensation produced by the matching hand either equals, doubles, or halves the size of the sensation produced by the target hand." The subjects were encouraged to make each match quickly, spontaneously, and independently of other matches. They received trial-by-trial instructions via a computer display projected by an LCD panel onto a screen $250 \mathrm{~cm}$ in front of them.

Each subject was required to generate a reference force with one hand followed by a ratio judgment with the opposite hand. The reference force was indicated on a null-meter display. A central, green LED indicated when the force was within $\pm 5 \%$ of the nominal target, and yellow and red LEDs on either side of the green LED indicated successively larger errors. The subject was required to keep the force within the target window for $1 \mathrm{sec}$, at which time a computer beep signaled the subject to relax. Immediately the word "Half," "Equal," or "Double" was presented on the screen, and the subject then made the required judgment with the opposite hand, pressing a footswitch when satisfied and then relaxing. The computer averaged and recorded the force measured for $0.2 \mathrm{sec}$ immediately prior to the switch press. If the coefficient of variation of the match force was greater than $5 \%$, the subject was required to repeat the trial. The subject was also given the option of skipping a trial during either the target or the match phase (usually if the required force was too large or too small to hold reliably) or choosing to repeat the trial during the match phase if he/she felt the judgment was incorrect. There was a $2-\sec$ delay between trials.

The subjects made matches to 14 different forces spaced from roughly $1 \%$ to $80 \%$ of their estimated MVC in equal ratio steps of 1.4. The experimenter estimated the MVCs on the basis of the subjects' physiques and then assigned the subjects to the following cat- egories: (1) maximum target force of $60 \mathrm{~N}, 2$ subjects; (2) maximum target force of $84 \mathrm{~N}, 3$ subjects; and (3) maximum target force of $118 \mathrm{~N}, 2$ subjects. If a subject was uncomfortable with the range of forces he/she was required to produce in the initial parts of the first session, the range was changed and the session restarted. While it would have been more straightforward to measure a subject's MVC prior to the first session, it was possible that making a maximal effort before the main experiment could have biased the subject's matching responses by providing the $\mathrm{MVC}$ as a reference or anchor point. Furthermore, the use of fixed ranges divided into equal-ratio steps guaranteed that all subjects were required to match at least some of the same target forces (e.g., the top 12 targets for one range were the same as the bottom 12 forces of the next higher range).

MVCs were measured at the completion of the entire experiment by asking the subjects to make maximal pinches alternately with the left and right hands, five times each. The five measurements were averaged geometrically to estimate the MVC. The MVC of the selfreported dominant hand was greater than that of the nondominant hand for all subjects. The guessed MVCs were correlated modestly but significantly with the geometric means of the actual MVCs for the two hands $\left(r^{2}=.50, p<.05\right)$ with a slope of 0.95 .

Once a range of forces was selected for a given subject, equating judgments were made for all 14 reference forces, halving judgments were made for the highest 11 forces, and doubling judgments were made for the lowest 11 forces. The targets were presented in blocks of 14 trials, with reference force and match ratio chosen at random. For all but 1 force in the equal judgments, each target was presented three times during the experiment. The remaining force, the 8th highest in the total range, was presented 21 times during the course of the session. The repetition was used to monitor fatigue throughout the session. Overall, the subjects performed 126 trials within a session.

The subjects completed four sessions, two each with the right hand as the reference and the left as the match and two in the opposite matching direction. Six subjects used the dominant hand as the reference in the first session. The hands were switched in the second session. The third and fourth sessions repeated the sequence of the first two.

Analysis. The data from each session were fit using two different empirical models. First, each of the three matching functions (half, equal, and double) were fit with individual power functions via weighted least squares regression (Bevington, 1969, p. 101), using means and standard errors calculated for each force target in log-log coordinates. The same data were also fit using a single-slope model, that is, a family of three parallel lines, one each for the three match ratios. The derivation of the single-slope model is given in the Appendix, and follows from assuming that the psychophysical function is a simple power function. The single-slope model was fit using an iterative Marquardt algorithm, again minimizing the weighted squared residuals. ${ }^{2}$

It is essential to apply both models and to compare their relative goodness of fit. If the three-slope model has a significantly better fit than the single-slope model, then the halving-doubling ratio is force dependent and the underlying psychophysical function is not a power function. If the single-slope model is valid, that is, its fit is not significantly worse than the three-slope fit, then the halving and doubling results can be summarized entirely by two ratios, namely, the force ratios that are perceived as half and double. The fits were compared using an $F$ test of the ratio of the sum-squared residuals.

\section{Results}

The halving, doubling, and equating judgments for all four sessions from 2 subjects are shown in Figures 2 and 3. The matches made from the nondominant hand (nd) to the dominant hand (d) are at the top of each figure, and the $\mathrm{d} \rightarrow$ nd matches are at the bottom. The first session of each pair is on the left. The data in Figure 2 have the worst overall fit to the single-slope model for any subject. The single- 
slope fit was also significantly worse $(p<.05)$ than the three-slope fit in two of the four sessions (marked by the asterisks). These were the only cases out of 28 such fits in the entire experiment where the single-slope model failed. The data in Figure 3 yield one of the better fits, and the one- and three-slope fits were not significantly different in any session.

Both sets of data illustrate several of the consistent findings across subjects. First, the slopes of all of the functions were less than 1 , indicating an overall compression bias or regression effect (for a review, see Poulton, 1989; see also S. S. Stevens \& Greenbaum, 1966; S. S. Stevens \& Guirao, 1963). It is important to note that a compression bias reduces both the slopes of the matching functions and the separation between them, thus corrupting the apparent matching ratios. To estimate the matching ratios accurately, the compression bias is modeled explicitly as a power function, with an exponent $\beta$, that modifies the reference sensation (Equations A4 and A10; also Van Doren, 1995a). The average value of $\beta$ across subjects ${ }^{3}$ was 0.71 , which confirms the observation that the bias is strongly compressive. The value of $\beta$ for individual subjects is listed in Table 1 .
The second trend is that the separations between the three functions are not symmetric. This is especially evident for the data in Figure 2, which shows a persistent bias toward larger ratios between doubled and equated forces than between equated and halved forces. The ratio of the differences in the intercepts can be used as a measure of asymmetry; that is,

$$
\rho=\frac{B_{2}-B_{1}}{B_{1}-\mathrm{B}_{1 / 2}}
$$

where the $B \mathrm{~s}$ are the intercepts of the halving, doubling, and equating functions. The asymmetry was consistent for each subject. In all four sessions, the values were $\rho>1$ for 3 subjects and $\rho<1$ for 2 subjects; in three of four sessions, the values for 2 subjects were $\rho<1$. Across subjects, however, the bias canceled out. The geometric mean of $\rho$ was 1.02 and was not significantly different from unity.

The differences between the intercepts, $B$, also give the first indication that the psychophysical function under these conditions is decelerating. The antilog of the difference between the intercepts gives the approximate ratio of matching forces that are perceived as half or twice the ref-

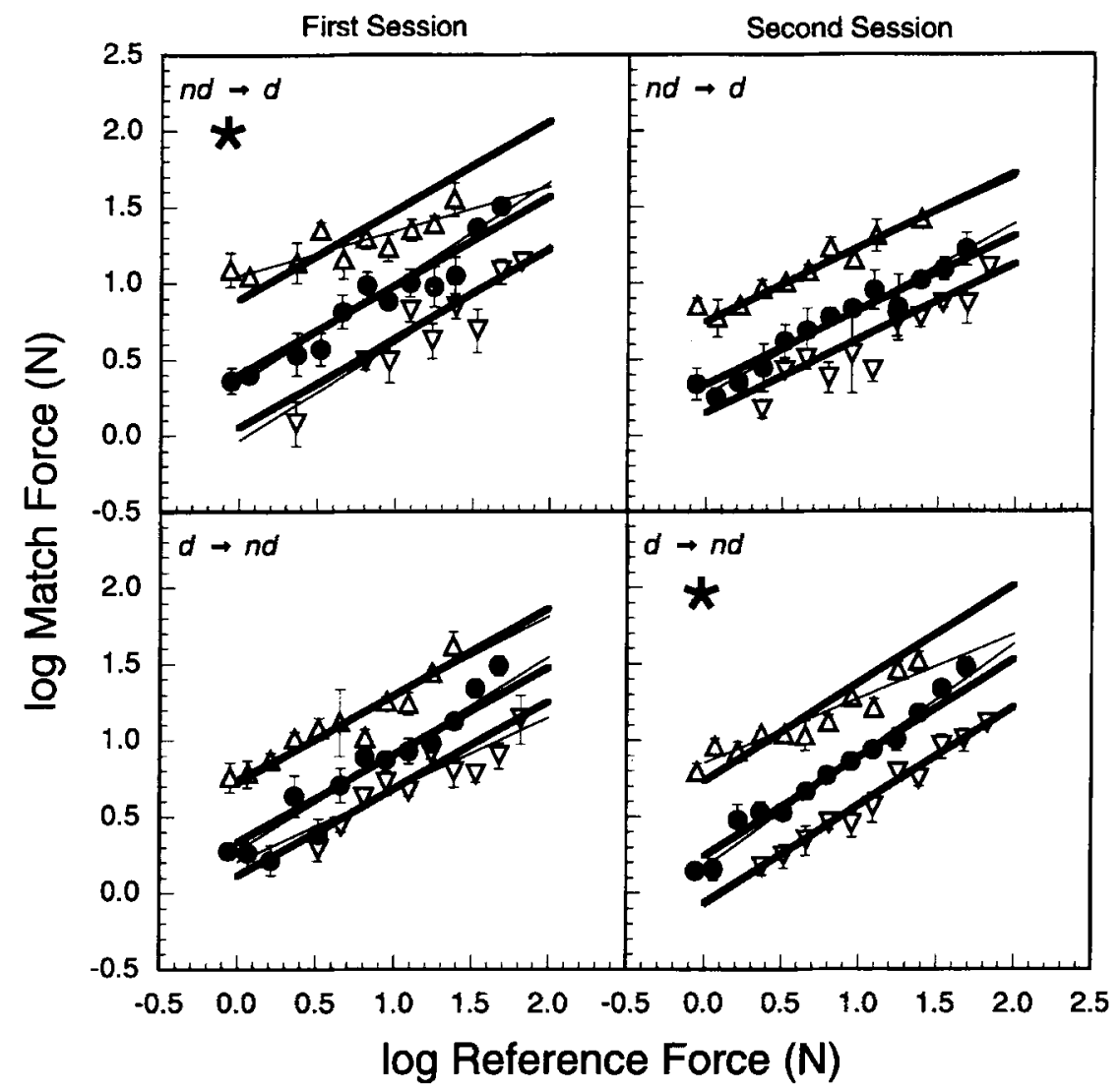

Figure 2. Average halving (downward triangles), equating (circles), and doubling (upward triangles) judgments made by 1 subject in four sessions. Each point is the average of three repetitions. Error bars are the standard errors of the mean. The thin lines are power functions fit to the individual functions. The thick lines have the same slope, and are the predictions of the halving-doubling model presented in the Appendix. The data shown here had the worst fit to the single-slope model, and the single-slope fit was significantly worse than the combined fit of the three individual power functions in two sessions (asterisks). 


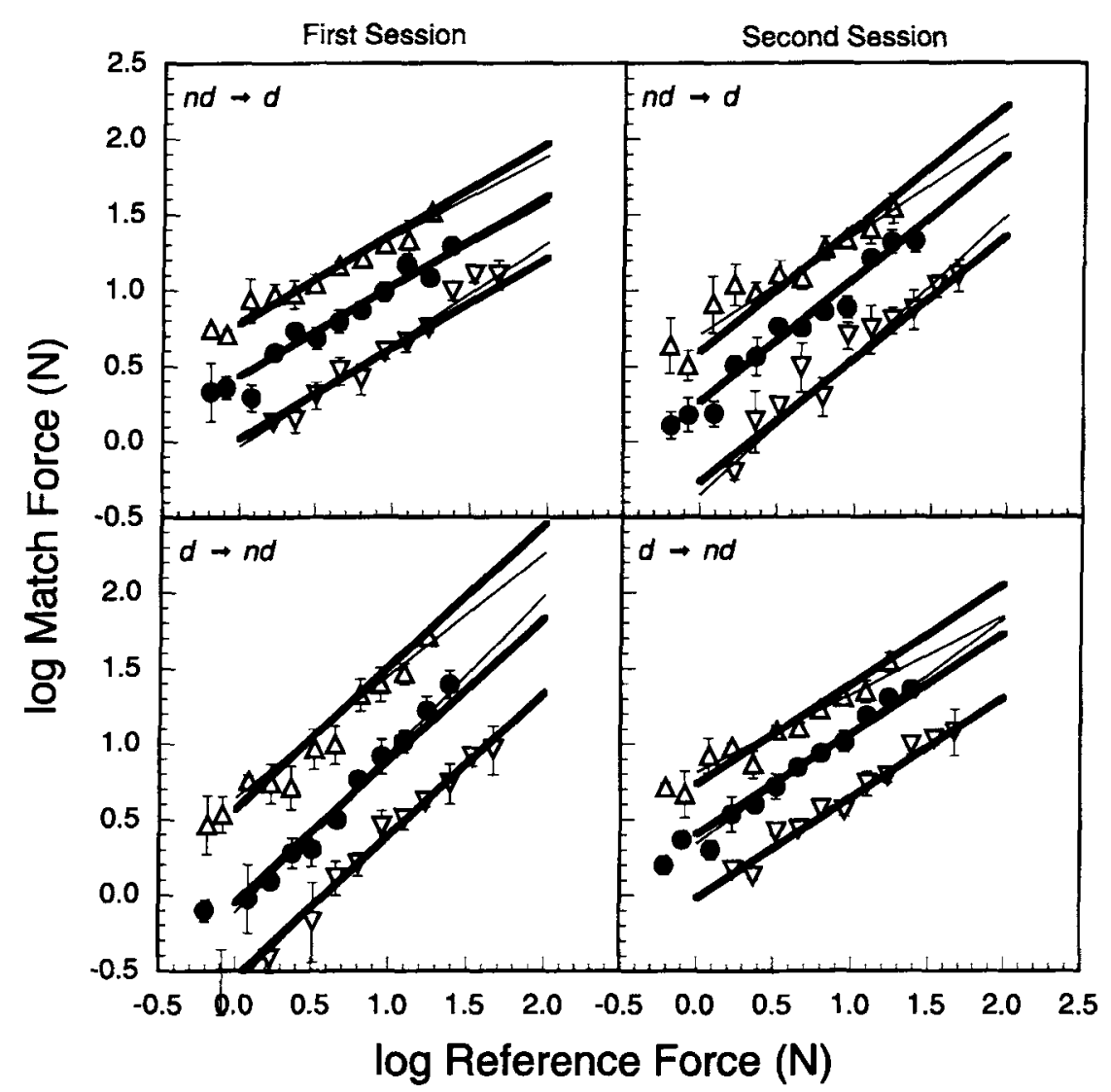

Figure 3. Data as in Figure 2, but for a subject fit well by the single-slope model. In this case, the single-slope model fit all of the sessions as well as the three-slope model.

erence force under the assumptions of the single-slope model. The ratios calculated from the intercepts of individual sessions ranged from 1.54 to $4.18,75 \%$ of them were greater than 2 , and the overall geometric mean ratios were 2.32 for halving and 2.34 for doubling. The average ratios for each subject are summarized in Table 1. A force ratio greater than 2 implies that a doubling of sensation requires more than a doubling of the force, consistent with a decelerating psychophysical function. As a second, very rough analysis, geometric means of the halving and doubling ratios within each session were calculated for each subject and each session. Note that these calculations are independent of any model. The ratios ranged from 1.74 to $4.88,85 \%$ of them were greater than 2 , and the overall geometric mean ratios were 2.43 for halving and 3.15 for doubling. Regardless of whether the analysis relies on the model or not, the great majority of the halving and doubling ratios were greater than 2 .

The ratio calculations from the intercepts are only approximate, however, since the effects of the compression bias were not accounted for. It is more accurate to calculate the exponents of the psychophysical functions from the relation between the intercepts and the nominal judgment ratios $(1 / 2,1,2)$. According to the model (Equation A11), the intercepts should be linearly related to the $\log$ of the nominal ratios. The slope of this function for each hand is equal to $\beta / \alpha$, where $\alpha$ is the exponent of the psychophysical function and $\beta$ is the bias exponent, as before. The assumption that the nominal ratios are $1 / 2,1$, and 2 requires that the matching functions be symmetric. Although that is not the case for individual subjects, it is true for the average results across subjects. The slope of the average halving-doubling function intercepts, plotted in Figure 4, yield decelerating psychophysical function exponents of $\alpha_{d}=0.59$ and $\alpha_{n d}=0.58$. Therefore, the force ratios resulting in sensations that differ by a factor of 2 (Equation A14) were 3.24 (dominant) and 3.33 (nondominant). Note that these ratios are larger than those predicted simply from the intercept differences because the latter are reduced by the compression bias.

Table 1

Summary Parameters of Single-Slope Fits for Individual Subjects

\begin{tabular}{lccccccc}
\hline & \multicolumn{7}{c}{ Subject } \\
\cline { 2 - 8 } \multicolumn{1}{c}{ Parameter } & 1 & 2 & 3 & 4 & 5 & 6 & 7 \\
\hline $\boldsymbol{\beta}$ & 0.75 & 0.75 & 0.73 & 0.61 & 0.57 & 0.73 & 0.85 \\
Halving ratio* $^{*}$ & 2.55 & 2.93 & 1.77 & 2.51 & 1.85 & 2.71 & 2.16 \\
Doubling ratio* $^{1.84}$ & 1.84 & 2.55 & 2.35 & 2.32 & 2.80 & 2.05 & 2.60
\end{tabular}

* Ratios are not corrected for compression bias. 


\section{EXPERIMENT 2 Unilateral Halving and Doubling}

Whether or not one uses the analytical models for halving and doubling and compression bias, the data from the first experiment imply that the psychophysical function of perceived force is decelerating. J. C. Stevens and Mack (1959), in contrast, report halving and doubling judgments consistent with an accelerating psychophysical function with an exponent of 2.1. Experiment 1 measured bimanual judgments of pinch force, whereas J. C. Stevens and Mack measured unimanual judgments of handgrip. Experiment 2 was performed to replicate J. C. Stevens and Mack's results and, if possible, to identify effects due to procedural differences.

\section{Method}

Subjects. Five female and 5 male subjects, aged 18-54 years (median 29), participated in the experiment. All subjects reported being right-handed, although the MVCs (see below) for 3 subjects were slightly higher for the left hand. J. C. Stevens and Mack (1959, p. 406) also used 10 right-handed subjects, although they were all male, "mostly students in the university."

Apparatus. The apparatus for the J. C. Stevens and Mack (1959) study and the present experiment are shown in Figure 5. In the J. C. Stevens and Mack experiment (Figure 5A), subjects squeezed nearly isometrically against a handle attached to a tensile force gauge (stiffness $=4.5 \times 10^{3} \mathrm{~N} / \mathrm{cm}$ ). The grasp posture was very similar in the present experiment (Figure 5B), with subjects squeezing a Jamar dynamometer, again nearly isometrically (stiffness $=6.1 \times 10^{3} \mathrm{~N} / \mathrm{cm}$ ). The dynamometer was calibrated prior to the experiment, and was read to the nearest $0.25 \mathrm{~kg}$ ( 1 quarter division).

The subjects sat with their forearms supported by a padded table (as in Experiment 1), with their hands extending over the edge of the table to accommodate the dynamometer. The dynamometer was held aloft by the subject, and supported partially by the experimenter

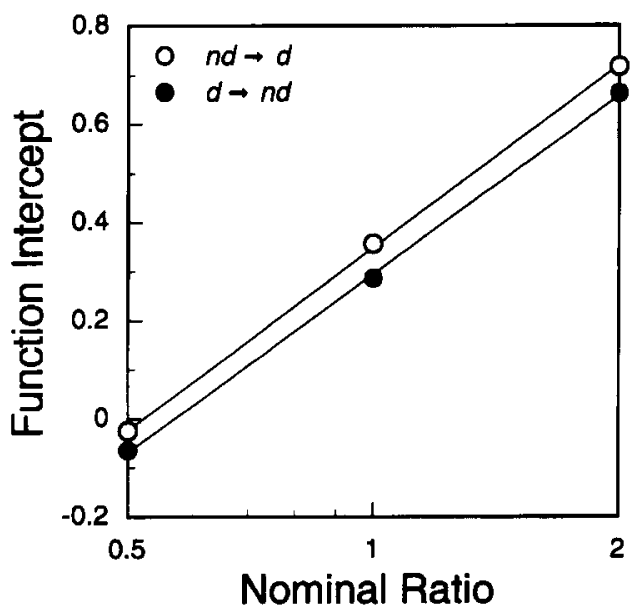

Figure 4. Intercepts of halving, equating, and doubling functions for both matching directions (nondominant-to-dominant and the converse) plotted as a function of the nominal judgment ratios. Points are from the average results across subjects. The halving-doubling model (Appendix) predicts that the intercepts will be a logarithmic function of the nominal ratios, as confirmed by the data. The slopes of the functions are equal to the ratio $\beta / \alpha$, where $\beta$ is the exponent of the compression bias function and $\alpha$ is the exponent of the psychophysical function. (in a manner that did not affect the force measurements). In the J. C. Stevens and Mack study, the force gauge was fastened to a table.

Procedure. The subjects were read written instructions in both experiments. The published (but paraphrased) instructions in the J. C. Stevens and Mack study (p. 406) were: "In this experiment you are asked to exert on a handle at various times a light, moderate, or heavy force, and then after releasing the handle to exert a force that seems to you one-half or twice as great. 'One-half' or 'twice' means what seems to you to feel like one-half or double the force. Before the experiment, you may pull the handle a few times to get accustomed to it. As you proceed, you will be told in each case whether to exert a moderate, light, or heavy pull, and also whether to halve or double it." The instructions for the present experiment were similar, except that "dynamometer" replaced "handle," and the subjects were told to hold each squeeze for about $1 \mathrm{sec}$ to allow the experimenter to read the dial. Also, these instructions were preceded by an introduction in which the subjects were cautioned to use forces that they felt they could double or halve (e.g., not to exert their maximum force on a "heavy" doubling trial), and not to try to repeat the same force in each category. The structure of the trials was also described (sets, blocks, timing, rest, etc.).

Subjects in the J. C. Stevens and Mack experiment made two sets of 15 judgments in three blocks of five trials each. Each set was completed using either halving or doubling judgments. The order of the sets was counterbalanced across subjects. The same protocol was used in the present experiment, except that subjects made judgments with both hands, so that each subject completed four sets of trials. The order of hand and judgment was randomized across subjects, with the exception that consecutive sets were never performed with the same hand. The introduction of left-hand judgments into the paradigm is the only major difference between the two experiments.

The subjects in the present experiment also made maximal squeezes at the end of the halving-doubling trials. They were asked to squeeze as hard as they could for $1 \mathrm{sec}$, to rest for $1 \mathrm{~min}$, and then to squeeze maximally with the opposite hand. The sequence was performed five times.

\section{Results}

The results for the left and right hands were analyzed separately following the general method used by J. C. Stevens and Mack (1959, p. 407). Those authors partitioned the reference forces into "six equal intervals of force" and calculated the median match-to-reference force ratio in each interval. Their results are replotted in Figure 6 (filled triangles), with the match ratio converted back to a matching force at each reference force. Since adjacent median reference forces are all separated by approximately $22-27 \mathrm{~N}$, it appears that the intervals were spaced linearly. The data from the present study were partitioned differently, with the reference forces divided into intervals with an equal number of observations (25). The results are plotted in Figure 6 (open symbols). Equal force intervals yielded similar results, but the number of observations in each interval varied widely - from 3 to 90 - generally decreasing with increasing force.

The slopes of the functions are quite similar $(0.91 \leq \beta \leq$ 0.95 ), but the separation between the present halving and doubling functions is much larger than the separation between the functions measured by J. C. Stevens and Mack (1959). As discussed above, the larger separation between the halving and doubling functions from the present experiment implies a smaller psychophysical exponent. The data from both experiments were fit using the one-slope model described above, and the parameters are listed in 

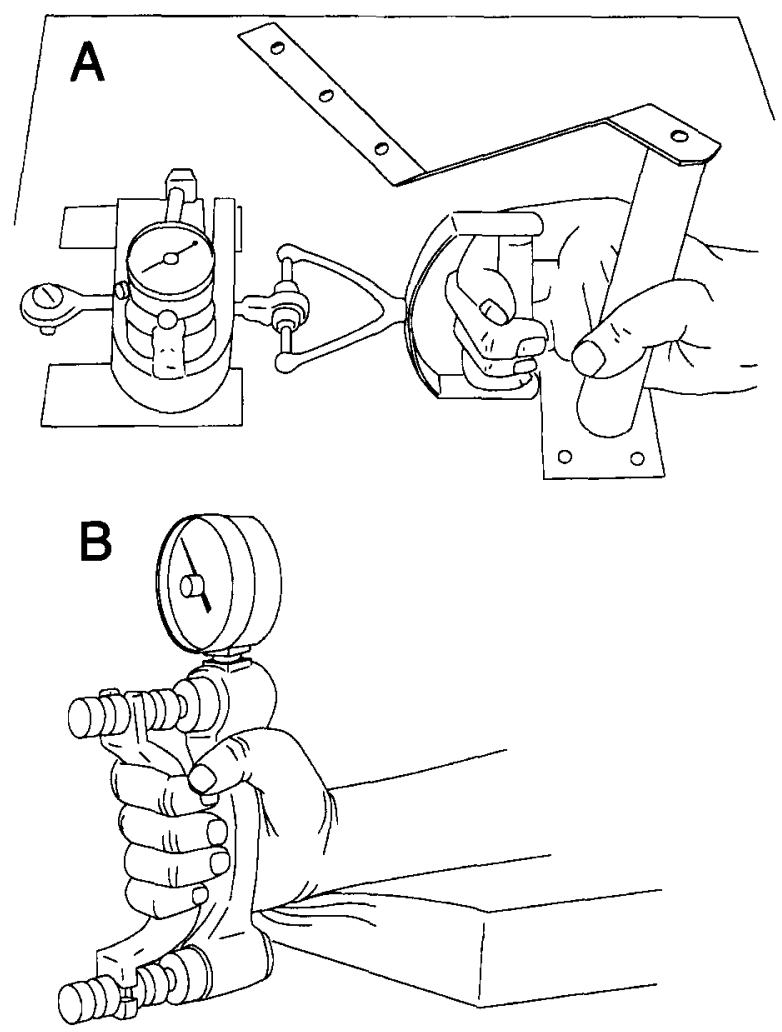

Figure 5. (A) Apparatus used in the isometric grip-force halvingdoubling experiment of J. C. Stevens and Mack (1959, redrawn from their Figure 1). Subjects pulled against a handle connected to a tension gauge. (B) Apparatus and hand posture used in Experiment 2. Subjects squeezed a Jamar dynamometer. Their forearms were supported by a cushioned table.

Table 2. Since only one hand was used at a time in each of these experiments, the bias model was used as in the bilateral experiment, but the parameters of the reference and match psychophysical functions were identical (see Equations A5-A7). The data from J. C. Stevens and Mack were fit best with a psychophysical exponent of $\alpha=2.0$ following correction for a compressive bias function with an exponent of $\beta=0.91$. The compression biases were similar in the present experiment (right hand, $\beta=0.95$; left hand, $\beta=0.91$ ), but the psychophysical exponents were decelerating rather than accelerating (right hand, $\alpha=$ 0.80 ; left hand, $\alpha=0.75$ ), consistent with, but slightly higher than, the bilateral halving and doubling exponents from Experiment 1.

\section{EXPERIMENT 3 \\ Pinch Compliance Characteristic}

The results of the first two experiments both suggest that perceived force is a decelerating function of actual force, even though the experiments used different grasps, manipulanda, paradigms, and subjects. Consequently, the small exponent cannot be attributed easily to methodological details of either experiment. What is most perplexing is that the second experiment closely approximated the conditions of the study by J. C. Stevens and Mack (1959) but yielded a strikingly different result.

Although the discrepancy between the studies cannot be explained satisfactorily, it is possible, at least, to offer a plausible basis for the present results that can be tested independently. Since kinesthesis occurs in parallel with the motor output generated by the subject, judgments can be based on afferent information or on the efferent command sent to the musculature (see the introduction to the present article). If judgments are made on the basis of the efferent command, then it is reasonable to expect a functional relationship between the putative sensory judgments (such as halving and doubling) and the physical properties of the effector responsible for generating the stimulus.

By way of analogy, we can suppose that a driver controls the speed of an automobile strictly by sensing depression of the accelerator pedal (a perception of the motor command) and not by looking at the speedometer (afferent feedback). If the driver doubles the perceived motor command by depressing the accelerator pedal twice as far, the actual change in the measured output (speed) depends on the nonlinear function relating engine power to pedal position and the resistance presented by the road. If an outside observer knows the resistance and the performance characteristics of the car, then the actual motorcommand judgments made by the driver (via the pedal) can be inferred from the measured velocity. Likewise, an adequate model of the relationship between the motor command to the hand and the resulting grasp output will

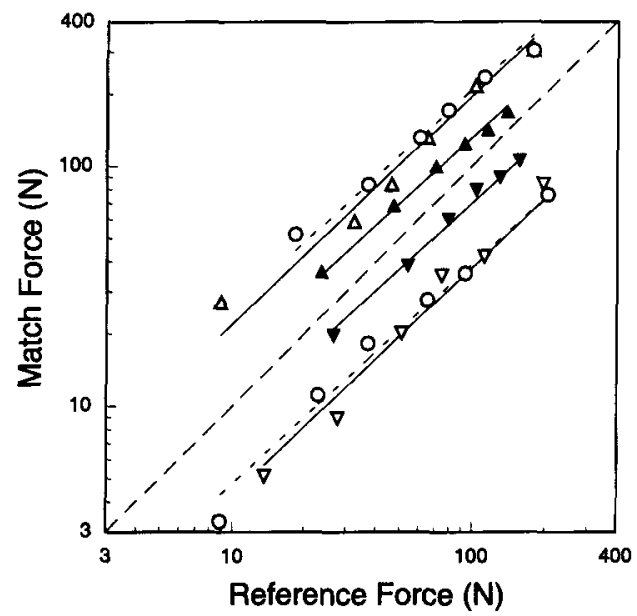

Figure 6. Grip-force halving and doubling results, with the matching force plotted as a function of the reference force. Halving data are below the diagonal, and doubling data are above the diagonal. Lines are best fits of the single-slope model made to each set of data. Open symbols are data from Experiment 2. Circles and dashed lines are from the right hand, and triangles and solid lines are data from the left hand. Each point is the median reference force and median matching force calculated from the median matching ratio within reference-force intervals containing 25 observations. Closed triangles are from J. C. Stevens and Mack (1959, adapted from their Figure 2). Each point represents the medians as for Experiment 2, but the intervals spanned equal linear ranges of the reference force rather than equal numbers of observations. 
Table 2

Parameters of Unimanual Halving and Doubling Fits

\begin{tabular}{lccr}
\hline & $\begin{array}{c}\text { J. C. Stevens \& } \\
\text { Mack (1959) }\end{array}$ & \multicolumn{2}{c}{ Present Study } \\
\cline { 3 - 4 } \multicolumn{1}{c}{ Parameter } & Right Hand & Right Hand & Left Hand \\
\hline Slope & 0.91 & 0.95 & 0.91 \\
Halving Intercept & 0.024 & -0.32 & -0.23 \\
Doubling Intercept & 0.30 & 0.40 & 0.50 \\
$\beta$ & 0.91 & 0.95 & 0.91 \\
$\alpha$ & 2.0 & 0.80 & 0.75 \\
\hline
\end{tabular}

allow us to relate hypothesized judgments of effort to measured forces.

The only model of motor control that has been used to specifically predict sensory judgments is the $\lambda$ model described by Feldman and his colleagues (e.g., Asatryan \& Feldman, 1965; Feldman, 1986; Feldman \& Latash, 1982). The model is sketched diagrammatically in Figure 7, and is one of a class of equilibrium-point models in which the final position and force of a limb result from the mechanical equilibrium between the load and the spring-like characteristics of the limb's muscles. To apply the model here, the thumb and fingers are treated as an effector with a single muscle squeezing a rigid load. The nonlinear stiffness of the muscle is described by an accelerating compliance characteristic (e.g., Asatryan \& Feldman, 1965), which will be expressed as:

$$
F=n x^{\varepsilon},
$$

where $F$ is the restoring force produced by an imposed stretch, $x$, given a fixed motor command. The motor command changes the rest length, $\lambda$, of the muscle via alteration of the stretch reflex threshold (see Latash, 1993, pp. 26-36, for a review). If $\lambda$ is set equal to the rest length of the load (point A in Figure 7), no force is generated. If $\lambda$ is shortened by $\Delta \lambda$ (point $B$ ), the unloaded muscle will contract to that shorter length (shown by the lightly drawn muscle). The load prevents muscle shortening, however, resulting in a force developed by the muscle (point $\mathrm{C}$ ) as though the muscle had been stretched by $\Delta \lambda$.

If we suppose that subjects halve and double the motor command $\Delta \lambda$ rather than the actual force, then we would expect the resulting force ratio to be:

$$
\frac{F(2 \Delta \lambda)}{F(\Delta \lambda)}=2^{\varepsilon}
$$

Equivalently, the inferred exponent of the psychophysical function (compare Equation 3 with Equation A14) would be given by:

$$
\alpha=\frac{1}{\varepsilon}
$$

For the bilateral pinch halving-doubling results from Experiment 1, the average exponents were 0.59 (dominant hand) and 0.58 (nondominant), yielding a predicted exponent, $\varepsilon$, of the compliance characteristic of about 1.7.

It is possible to test this prediction directly by physically measuring the compliance characteristic for three-finger pinch. Such measurements are typically made by using force or displacement step perturbations of a subject's limb while the subject maintains a steady effort (motor command) and is instructed to "not intervene" (i.e., change the command) when the perturbation is applied. Perturbation measurements of this type have been made previously at individual joints such as the elbow (e.g., Asatryan \& Feldman, 1965), the distal interphalangeal joint of the thumb (Carter, Crago, \& Keith, 1990), and the ankle (Gottlieb \& Agarwal, 1980), but not for three-finger pinch. The purpose of the third experiment was to make such measurements, using an active, pneumatic manipulandum, and then to compare the measured compliance characteristic with the predictions from the halving-doubling results.

\section{Method}

Compliance characteristics were measured on 1 female and 5 male subjects, aged 24-36 years, with no reported sensorimotor abnormalities.

The manipulandum, shown in Figure 8, consisted of two pneumatic cylinders driven by a common air supply that could be switched electronically between two manually set pressures. The two fingers contacted the upper bar connecting the moving pistons of the cylinders, and the thumb contacted the lower bar connecting the cylinder bodies. The thumb pressed on a load cell via a bearing-mounted piston. Span was measured by using an optical encoder. With air pressures up to $90 \mathrm{psi}$, the device was capable of producing forces in excess of $60 \mathrm{~N}$.

The manipulandum was held aloft in the orientation shown in Figure 8 , with the weight of the device supported by the thumb. Prior to each trial, the thumb force was measured without an applied fin-



Figure 7. Equilibrium point model of pinch. The thumb and fingers are modeled as a single effector with a muscle that has a nonlinear compliant characteristic. The thumb and finger squeeze a rigid external load. Initially, the rest length, $\lambda$, of the effector is equal to the length of the external load (point $A$ ), so no force is exerted. Changing the efferent command shifts the rest length by $\Delta \lambda$ (point $B$ ). If the muscle was unloaded, it would shorten to the new length (lightly drawn muscle). The load prevents shortening, however, so the muscle exerts a force, $F$, against the load. The force is at the point of equilibrium between the effector and load (point $\mathrm{C}$ ). 
ger force, and this offset force was subtracted from subsequent measurements. The subjects squeezed the device to a preset span indicated by a mark on the cylinder shaft (approximately $4 \mathrm{~cm}$ ), and the pressure to the air cylinders was switched from low to high at irregular times between 1 and $1.5 \mathrm{sec}$ after the subject had reached the target span. The subjects were instructed not to intervene in response to the perturbation. ${ }^{4}$ Forces and spans were recorded at a sampling rate of $150 \mathrm{~Hz}$, and the data $500 \mathrm{msec}$ just prior to the perturbation and between 500 and $1,000 \mathrm{msec}$ following the perturbation were collected and averaged. The change in force divided by the change in span was taken as the stiffness at the arithmetic mean of the initial and final forces.

\section{Results}

The time courses of the force and span are plotted in Figure 9 for one trial, in which the force (lower trace) and span (upper trace) held steady prior to the perturbation and reached a stable plateau after perturbation, consistent with the do-not-intervene paradigm (e.g., Crago, Houk, \& Hasan, 1976). The brackets mark the data averaged for the trial. Trials that did not have stable plateaus were rejected from the analysis. Each subject completed between 30 and 54 trials at six to eight pressure settings, depending on their strength. Of these trials, 11-37 were successful based on attainment of a stable plateau.

Data for one representative subject are shown in Figure 10 (open circles), where the stiffness is plotted as a function of the average force. There is appreciable scatter, but the modest correlation $\left(r^{2}=.46\right)$ was highly significant $(p=.0002)$. The heavy line in Figure 10 shows the least squares fit to the data. The thin lines are fits to the data from the other 5 subjects. Five subjects had a significant positive correlation between stiffness and force $(p<.03)$. The

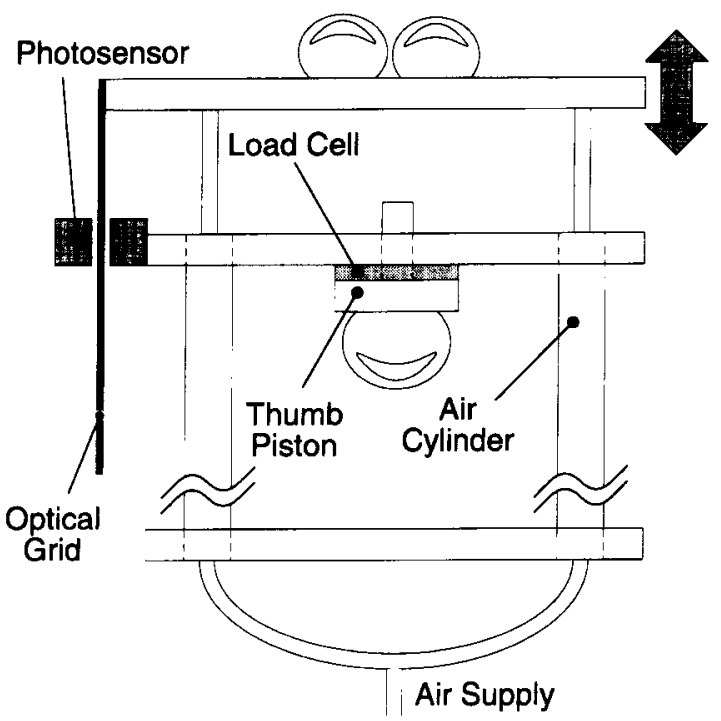

Figure 8. Pneumatic manipulandum used to deliver force perturbations in Experiment 3. The manipulandum consists of two pneumatic cylinders driven by a common air supply. A pair of manually adjusted regulators and solenoid valves allowed the cylinders to be switched electronically between two pressures, providing a step increase in the force exerted on the hand. The pinch force was measured by the load cell under the thumb, and span was measured by a custom-built optical encoder.

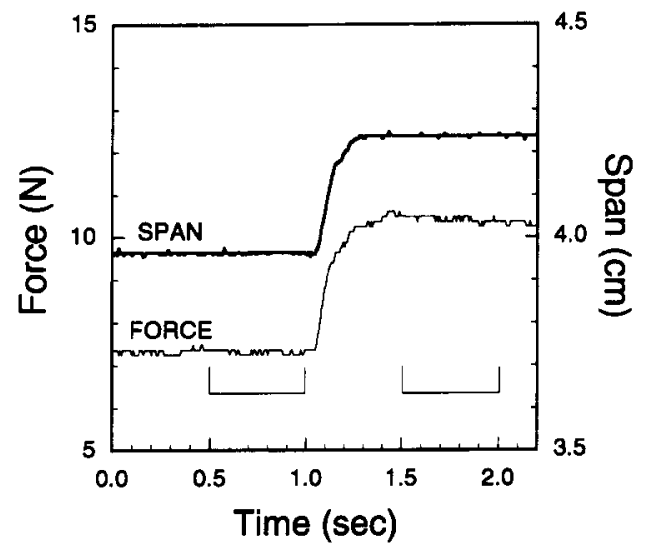

Figure 9. Time courses of the span and force measured during a perturbation trial. After the subject reached the target span and held it steady, the pressure was switched from low to high at an unpredictable time, resulting in an increase in both the span and pinch force. The stable plateaus before and after the perturbation were required for acceptance of the trial. The brackets show the times over which the data were averaged to calculate the stiffness, given by the change in force divided by the change in span.

subject who had only 11 successful trials had a correlation that just failed to reach significance $\left(r^{2}=.302, p=.08\right)$.

The predicted stiffness, $K$, as a function of force can be calculated by differentiating Equation 2 and then making a variable substitution of $F$ for $x$, yielding

$$
K(F)=\frac{\mathrm{d} F}{\mathrm{~d} x}=\varepsilon n^{1 / \varepsilon} F^{(\varepsilon-1) / \varepsilon} .
$$

The exponents of the power functions fit to the stiffnesses (Figure 10) ranged from 0.29 to 1.0 , with an average value of 0.48 , yielding an exponent of $\varepsilon=1.9$ for the compliance characteristic. The accelerating compliance characteristic, in turn, implies a decelerating psychophysical function (Equation 4) with an exponent of $\alpha=0.53$, in good agreement with the bilateral halving-doubling results.

\section{DISCUSSION}

The results of the two halving-doubling experiments imply directly that the psychophysical function of "perceived force" is decelerating with an exponent between 0.6 and 0.8 . If we assume that subjects made judgments of the efferent command rather than of the force, then it is possible to account for the halving-doubling results using an equilibrium-point model of motor control. The critical link between the equilibrium-point model and the sensory judgments is the mechanical compliance of the hand, and the compliance derived from the halving-doubling data agreed well with that measured directly in the third experiment.

A similar association between kinesthetic judgments and motor control was made in an earlier experiment (Van Doren, 1995b), in which subjects matched the force of threefinger pinch while squeezing compliant or rigid loads with the two hands. Overall, that experiment closely replicated the results of a very similar study by Mai, Schreiber, and 


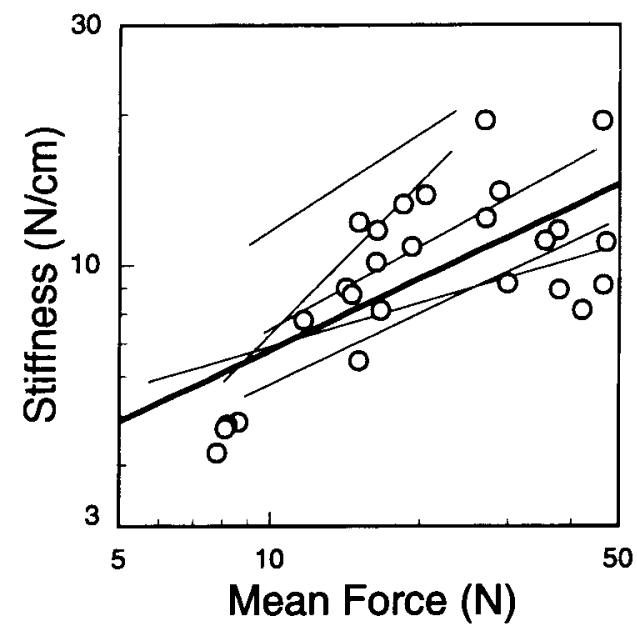

Figure 10. Stiffnesses measured as a function of the background force (given by the arithmetic mean of the initial and final forces). The points are the individual trials from a representative subject who had an intermediate value of the correlation coefficient $(0.50)$. The thick line is the least squares fit to that subject's data, and the thin lines are the fits to the data from the other 5 subjects.

Hermsdörfer (1991). Furthermore, the errors subjects made when the loads were different were consistent with the same equilibrium-point model used here, and predicted an accelerating compliant characteristic with an exponent of 1.5. The paradigm used in that experiment made it very difficult to make matches on the basis of afferent force information, since subjects were given a short time in which to make the match $(1 \mathrm{sec})$ and were not told which hand the reference or match was on any given trial. In other words, there was little time to make adjustments prompted by a sensory mismatch, and the subject would not know which hand required adjustment. As a result, it is reasonably certain that subjects resorted to matching the efferent commands to the two hands.

A plausible model exists, then, for the ratio judgments made under the conditions of this study. The assumption that subjects judged effort rather than force is consistent with most previous scaling and matching studies (e.g., Jones, 1986). In fact, those studies suggest that subjects naturally make effort judgments unless specific precautions are taken, such as detailed instructions to disregard effort (McCloskey et al., 1974; Roland \& LadegaardPedersen, 1977).

The observed psychophysical exponents ( 0.6 to 0.8 ), however, are at odds with previous halving-doubling and magnitude-estimation results-at least those that did not use the MVC as a modulus. All of those studies, as noted by J. C. Stevens $(1989,1974)$, found exponents of about 1.7 .

Why are the exponents so different? The discrepancy probably is not a result of subject differences, since subjects' halving and doubling judgments in Experiment 1 were fairly stable (see Figures 2 and 3 ) and the ratios were greater than 2 in at least $75 \%$ of the 56 halving or doubling functions ( 7 subjects, 4 sessions, 2 ratios per session). Few points had to be discarded as mistakes. More importantly, different subjects yielded corroborating results in Experiment 2 in which a different paradigm was used. It seems unlikely that the present data deviate from previous results by chance or subject selection.

Could the discrepancy instead be due to a systematic bias induced by the experimental paradigm? The possibility seems unlikely. There were, certainly, significant procedural differences between Experiment 1 and J. C. Stevens and Mack's (1959) study. In the latter, unilateral judgments of handgrip were used, whereas bilateral judgments of three-finger pinch were made in Experiment 1. The possibility of a spurious, though consistent, bias induced by the protocol is virtually eliminated by the results of Experiment 2 . Apart from making judgments with both hands (in separate sets) rather than with the right hand alone, the paradigm used in the second experiment was nearly identical to that used by J. C. Stevens and Mack-yet the results were markedly different.

Were the subjects in the different studies judging different continua? The discrepancy could be resolved potentially if the scaling judgments that resulted in accelerating psychophysical functions were made on the basis of afferent force information. The different exponents would then reflect differences in the scaled continua. The possibility, though, seems weak. J. C. Stevens and Cain (1970) measured accelerating magnitude-estimation functions even though the estimates made at a given force were systematically elevated by fatigue - the hallmark of effort judgments. Eisler (1962) also seems to have evoked both force and effort judgments in his matching experiments (see the introduction to the present article), but the magnitudeestimation and production functions were all accelerating.

Additional work is clearly needed to resolve this dilemma. First, the effects of instructions on scaling judgments need to be investigated systematically under consistent conditions. Although, in Experiment 2, an attempt was made to copy the published instructions of the earlier study by J. C. Stevens and Mack (1959), it was not possible to duplicate the incidental instructions that subjects inevitably receive in the course of an experiment. During preliminary trials of the force-matching experiment mentioned above (Van Doren, 1995b), 2 subjects interpreted the "match-force" instruction literally, and were able to generate equal forces with both hands in spite of asymmetric loads. The subjects acknowledged the difficulty of making such matches and reported using cutaneous sensory cues. Their behavior would have been encouraged in a "force" matching experiment, but discouraged in an "effort" matching experiment. Magnitude estimation is sensitive in general to instructions (e.g., Gescheider, 1988), and the problem is exacerbated here by the lability of the force-effort partiality. Second, the results described above reinforce the importance of the mechanical properties of the effector in accounting for putative sensory judgments, as proposed by Feldman and Latash (1982) and used by Van Doren (1995b). Ideally, compliance characteristics would be measured at the same time that scaling or matching judgments were made. It would be interesting, indeed, if the hand compliance could be measured in conjunction 
with scaling judgments made under conditions in which the subject reliably used afferent force information. Under such circumstances, the link between sensory judgment and motor control would be broken and we would not necessarily expect agreement between the compliance characteristics and the psychophysical functions. Sensorimotor experiments of this type offer fertile ground for exploration of the relationships that must exist between kinesthesis and motor control.

\section{REFERENCES}

Asatryan, D. G., \& Feldman, A. G. (1965). Functional tuning of the nervous system with control of movements or maintenance of a steady posture: I. Mechanographic analysis of the work of the joint on execution of a postural task. Biophysics, 10, 925-935.

BANISTER, E. W. (1979). The perception of effort: An inductive approach. European Journal of Applied Physiology, 41, 141-150.

BEvington, P. R. (1969). Data reduction and error analysis for the physical sciences. New York: McGraw-Hill.

CAFarelli, E., \& Bigland-RitchIE, B. (1979). Sensation of static force in muscles of different length. Experimental Neurology, 65, 511-525.

Carter, R. R., Crago, P. E., \& Keith, M. W. (1990). Stiffness regulation by reflex action in the normal human hand. Journal of Neurophysiology, 64, 105-118.

COOPER, D. F., Grimby, G., Jones, D. A., \& EdWARDS, R. H. T. (1979) Perception of effort in isometric and dynamic muscular contraction. European Journal of Applied Physiology, 41, 173-180.

Crago, P. E., Houk, J. C., \& Hasan, Z. (1976). Regulatory actions of human stretch reflex. Journal of Neurophysiology, 39, 925-935.

EISLER, H. (1962). Subjective scale of force for a large muscle group. Journal of Experimental Psychology, 64, 253-257.

EISLER, H. (1965). The ceiling of psychophysical power functions. American Journal of Psychology, 78, 506-509.

Feldman, A. G. (1986). Once more on the equilibrium-point hypothesis $(\lambda$-model) for motor control. Journal of Motor Behavior, 18, 17-54.

FeldMAN, A. G., \& LATASH, M. L. (1982). Afferent and efferent components of joint position sense: Interpretation of kinaesthetic illusions. Biological Cybernetics, 42, 205-214.

Gandevia, S. C., \& Burke, D. (1992). Does the nervous system depend on kinesthetic information to control natural limb movements? Behavioral \& Brain Sciences, 15, 614-632.

Gandevia, S. C., \& MCCloskeY, D. I. (1977). Changes in motor commands as shown by changes in perceived heaviness, during partial curarization and peripheral anesthesia in man. Journal of Physiology, 272, 673-689.

Gescheider, G. A. (1988). Psychophysical scaling. Annual Review of Psychology, 39, 169-200.

Gottlieb, G. L., \& Agarwal, G. C. (1980). Response to sudden torques about the ankle in man: II. Postmyotatic reactions. Journal of Neurophysiology, 43, 86-101.

Hellman, R. P., \& Zwislocki, J. J. (1961). Some factors affecting the estimation of loudness. Journal of the Acoustical Society of America, 33, 687-694.

JONES, L. A. (1986). Perception of force and weight: Theory and research. Psychological Bulletin, 100, 29-42.

JONES, L. A, \& HUNTER, I. W. (1982). The relation of muscle force and EMG to perceived force in the human finger flexors. European Journal of Applied Physiology, 50, 125-131.

JONES, L. A., \& HUNTER, I. W. (1983a). Effect of fatigue on force sensation. Experimental Neurology, 81, 640-650.

Jones, L. A., \& HunTER, I. W. (1983b). Perceived force in fatiguing isometric contractions. Perception \& Psychophysics, 33, 369-374.

Latash, M. L. (1993). Control of human movement. Chicago: Human Kinetics.

MaI, N., Schreiber, P., \& Hermsdörfer, J. (1991). Changes in perceived finger force produced by muscular contractions under isometric and anisometric conditions. Experimental Brain Research, 84, 453-460.

Matthews, P. B. C. (1982). Where does Sherrington's "muscular sense" originate? Muscles, joints, corollary discharges? Annual Review of Neuroscience, 5, 189-218.

McCloskey, D. I., Ebeling, P., \& Goodwin, G. M. (1974). Estimation of weights and tensions and apparent involvement of a "sense of effort." Experimental Neurology, 42, 220-232.

POULToN, E. C. (1989). Bias in quantifying judgments. Hillsdale, NJ: Erlbaum.

Roland, P. E., \& LadegaArd-Pedersen, H. (1977). A quantitative analysis of sensations of tension and kinaesthesia in man: Evidence for a peripherally originating muscular sense and for a sense of effort. Brain, 100, 671-692.

STEVENS, J. C. (1974). Psychophysical invariances in proprioception. In F. A. Geldard (Ed.), Cutaneous communication systems and devices (pp. 73-77). Austin, TX: Psychonomic Society.

SteVens, J. C. (1989). Static and dynamic exertion: A psychophysical similarity and dissimilarity. In G. Ljunggren \& S. Dornic (Eds.), Psychophysics in action (pp. 81-93). Berlin: Springer-Verlag.

Stevens, J. C., \& CaIN, W. S. (1970). Effort in isometric muscular contractions related to force level and duration. Perception \& Psychophysics, 8, 240-244.

STEVENS, J. C., \& MACK, J. D. (1959). Scales of apparent force. Journal of Experimental Psychology, 58, 405-413

Stevens, S. S., \& GaLANTER, E. H. (1957). Ratio scales and category scales for a dozen perceptual continua. Journal of Experimental Psychology, 54, 377-411.

Stevens, S. S., \& Greendaum, H. B. (1966). Regression effect in psychophysical judgments. Perception \& Psychophysics, 1, 439-446.

Stevens, S. S., \& Guirao, M. (1963). Subjective scaling of length and area and the matching of length to loudness and brightness. Journal of Experimental Psychology, 66, 177-186.

VAN DOREN, C. L. (1993). Individual differences in perceived pinch force and bite force. Perception \& Psychophysics, 53, 483-488.

VAN DOREN, C. L. ( 1995a). Cross-modality matches of finger span and line length. Perception \& Psychophysics, 57, 555-568.

VAN DOREN, C. L. (1995b). Pinch force matching errors predicted by an equilibrium point model. Experimental Brain Research, 106, 488-492.

\section{NOTES}

1. J. C. Stevens $(1974,1989)$ did not report that Eisler (1962) measured two exponents for leg presses. When the subject had to hold the leg up against the footpedal, the magnitude-estimation exponent was 1.5 . When the heel was supported by a strap, the exponent declined slightly to 1.3 .

2. There were two problems with the curve fitting that required prior processing. First, it seemed prudent to try to exclude the points where subjects may have erred in making halving or doubling judgments by doing the opposite (e.g., forgetting the instruction and halving instead of doubling). It seems clear that this must have happened for some points, because the halving judgments were, for example, greater than some of the doubling judgments for the same target force. Points were eliminated if halving judgments were greater than the target and if doubling judgments were less than the target. Of the 3,507 matching trials, only 122 $(3.5 \%)$ were excluded as mistakes.

The second problem was that the variance calculated for a set of matches at a particular reference force was occasionally very smallsmaller by more than an order of magnitude than the average variancewhich distorted the weighted fits. An $F$ test was used to compare the variance at each point with the pooled estimate of the variance from a data set (e.g., halving). If the ratio was too small $(p<.05)$, the pooled variance was substituted for the point variance. Of the 980 variances calculated, $5.5 \%$ were too small (consistent with the criterion significance level). Large $F$ ratios were not modified since such points would contribute little to the weighted fits. For points where there was only one trial to average, a standard error could not be defined, and these points were excluded from the fit.

3. The average used here is the tangent of the average arctangent of the exponents. Using the arctangents of the exponents (i.e., the angles corresponding to the slopes of the power functions in logarithmic coordinates) allows for well-defined arithmetic averaging. For example, the average of the slope arctangents of a horizontal line and a vertical line-that is, the average of $0^{\circ}$ and $90^{\circ}-$ is $45^{\circ}$, yielding an "average" slope of 1 . 
The arithmetic average of the raw slopes is infinite, and the geometric average slope is undefined.

4. While they squeezed the pneumatic manipulandum, the subjects also squeezed, in the opposite hand, one of the passive manipulanda used in the first experiment (Figure 1). Attending to the passive manipulandum made it easier for most subjects to disregard the perturbation by the pneumatic manipulandum.

\section{APPENDIX}

\section{Halving-Doubling Model With Compression Bias}

We start with psychophysical functions for both hands (dominant, $\mathrm{d}$, and nondominant, nd), each with a scale factor $k$ and an exponent $\alpha$ :

$$
\Psi_{\mathrm{d}}=k_{\mathrm{d}} F^{\alpha_{\mathrm{d}}}
$$

and

$$
\Psi_{\mathrm{nd}}=k_{\mathrm{nd}} F^{\alpha_{\mathrm{nd}}} .
$$

The task of the subject is to generate a reference force with one hand and then make a force with the opposite hand that is some ratio $m=\{1 / 2,1,2\}$ relative to the reference. If the matches are unbiased, then

$$
\Psi_{\text {match }}=m \Psi_{\text {ref }}
$$

However, well-known compression or centering biases that distort the match producing an actual matching sensation will be modeled as

$$
\Psi_{\text {match }}^{*}=b\left(\Psi_{\text {match }}\right)^{\beta}=b m^{\beta} \Psi_{\text {ref }}^{\beta},
$$

where the asterisk represents the biased sensation. Substituting in the expressions for the psychophysical function yields the expected matching function

$$
F_{\text {match }}=\left(\frac{b m^{\beta} k_{\text {ref }}^{\beta}}{k_{\text {match }}}\right)^{\frac{1}{\alpha_{\text {match }}}} F^{\beta \alpha_{\text {ref }} / \alpha_{\text {match }}}
$$

The biased matching functions (one for each ratio $m$ ), then, are also power functions. In $\log$ - log coordinates, the family of functions should be a set of three parallel lines with slope $\mu$ and intercepts $B_{j}$ given by

$$
\mu=\frac{\beta \alpha_{\text {ref }}}{\alpha_{\text {match }}}
$$

and

$$
B_{j}=\log \left[\left(\frac{b m_{j}^{\beta} k_{\mathrm{ref}}^{\beta}}{k_{\mathrm{match}}}\right)^{\frac{1}{\alpha_{\mathrm{match}}}}\right]
$$

There will be a set of such curves for both matching directions, that is, matching the dominant hand to the nondominant hand and conversely. The exponent of the bias function, $\beta$, and the ratio of the unbiased exponents, $\alpha$, can be calculated from the two slopes $\mu_{\mathrm{d} \rightarrow \mathrm{nd}}$ and $\mu_{\mathrm{nd} \rightarrow \mathrm{d}}$ :

$$
\mu_{\mathrm{d} \rightarrow \mathrm{nd}}=\frac{\beta \alpha_{\mathrm{nd}}}{\alpha_{\mathrm{d}}}, \mu_{\mathrm{nd} \rightarrow \mathrm{d}}=\frac{\beta \alpha_{\mathrm{d}}}{\alpha_{\mathrm{nd}}}
$$

so that

$$
\frac{\alpha_{\mathrm{d}}}{\alpha_{\mathrm{nd}}}=\sqrt{\frac{\mu_{\mathrm{nd} \rightarrow \mathrm{d}}}{\mu_{\mathrm{d} \rightarrow \mathrm{nd}}}}
$$

and

$$
\beta=\sqrt{\mu_{\mathrm{nd} \rightarrow \mathrm{d}} \mu_{\mathrm{d} \rightarrow \mathrm{nd}}} .
$$

It is of greater interest, perhaps, to get estimates of the exponents, $\alpha$, individually for each hand. To do so, we must make some assumptions about the values of the ratios, $m_{j}$. If we assume that these ratios are equal to their nominal values, then we can plot the intercepts, $B_{j}$ (three each for both matching directions) as a function of the $m_{j}$ :

$$
B_{(\mathrm{nd} \rightarrow \mathrm{d}) j}=\frac{1}{\alpha_{\mathrm{nd}}} \log \left(\frac{b k_{d}^{\beta}}{k_{\mathrm{nd}}}\right)+\left(\frac{\beta}{\alpha_{\mathrm{nd}}}\right) \log m_{j}
$$

and similarly for the $\mathrm{d} \rightarrow$ nd matches. The slopes, $M$, of these functions can then be used with the value of $\beta$ calculated above to get $\alpha_{\mathrm{d}}$ and $\alpha_{\text {nd }}$ :

$$
M_{\mathrm{d} \rightarrow \mathrm{nd}}=\frac{\beta}{\alpha_{\mathrm{d}}}, M_{\mathrm{nd} \rightarrow \mathrm{d}}=\frac{\beta}{\alpha_{\mathrm{nd}}}
$$

and

$$
\alpha_{\mathrm{d}}=\frac{\beta}{M_{\mathrm{d} \rightarrow \mathrm{nd}}}, \alpha_{\mathrm{nd}}=\frac{\beta}{M_{\mathrm{nd} \rightarrow \mathrm{d}}} .
$$

The averages of the parameters across subjects yields $\beta=0.710$, $M_{\mathrm{d} \rightarrow \mathrm{nd}}=1.21$, and $M_{\mathrm{nd} \rightarrow \mathrm{d}}=1.23$, giving $\alpha_{\mathrm{d}}=0.589$ and $\alpha_{\text {nd }}=0.576$.

The force ratio required to produce a twofold change in sensation is given by

$$
\frac{F_{2}}{F_{1}}=2^{\frac{1}{\alpha}}
$$

yielding halving-doubling force ratios of 3.24 for the dominant hand and 3.33 for the nondominant hand.

(Manuscript received October 7, 1994; revision accepted for publication August 28, 1995.) 